\title{
COUPLING BEAM DESIGN WITH SPECIAL MOMENT FRAME AND SPECIAL REINFORCED CONCRETE SHEAR WALLS
}

\author{
Agyanata Tua, MUNTHE \\ Faculty of Engineering \\ University Mercu Buana Jakarta, Indonesia \\ agyanata.umb@gmail.com \\ Muklish, NALAHUDDIN \\ Faculty of Engineering \\ University Mercu Buana Jakarta, Indonesia \\ muklishnalahuddin@gmail.com
}

\begin{abstract}
An Earthquake is on of the natural phenomena that cannot be avoided or cannot be prevented by its appearance which is very difficult to accurately predict both from the time and place of its occurrence. Shear wall system is used to increase the sitffness of many multi-storey building, in this case building that have more than 20 floors. Building structures with shear wall as retaining element of lateral force generally have good performance during an earthquake. Coupling beam is an connecting beam betweem two shear walls, this beam makes a series of shear walls works as a system that is able to withstand earthquake force. Coupling beam also make the working structure rigid and absorbs energy due to the very high rigidity of the coupling beam with shear wall behaving link two free cantilevers. Coupling beam is considered to be able to transmit shear force from one wall to another so that it can withstand large structural deformation. Structure design material strenght for concrete fc' $35 \mathrm{MPa} \sim$ fc 55'MPA and rebar (D10 \& D13) using fy 520MPa and fy 420MPa for diameter $>16 \mathrm{~mm}$. While the regulations used are SNI 1726: 2012, SNI 1727: 2013, and SNI 2847: 2013. Structural loading is given according to loading rules which are then analyzed using ETABS 2016 software.
\end{abstract}

Keywords: Earthquake ; Special Moment Frame ; Reinforced Concrete Shear Walls ; Coupling beam

\section{INTRODUCTION}

Indonesia is a country that has a high level of vulnerability to earthquakes, with geographical condition in parts of Indonesia within the Ring of Fire series where there are still many active volcanoes and the spread of areas that have faults and active tectonic plate faluts that can trigger earthqueake occurrence. This can be seen in various natural disasters that hit several region of Indonesia ranging from low, medium to high magnitudes. This condition requires the building designer to meet the criteria for building structure design to be able to withstand earthquake force with the aim at the time of the eartquake, the structure is able to privide security to building users from the risk of eartqueake hazard. 
Figure 1. map of the earthquake area of Indonesia

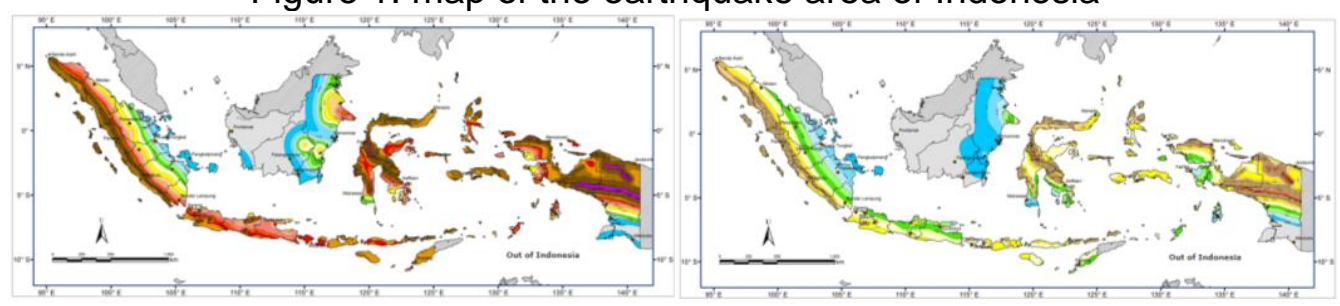

Source : Indonesia Hazard Book, 2017

Shear wall system is used to increase the stiffness of multi-storey buildings. In this case builing that have more than 20 floors. Building structure with shear walls as a lateral retaining element genereally have good performance during an earthquake, with the slightest damage occuring to the shear wall structural system during an earthquake. Coupling beam is a connecting beam between two shear walls, this beam makes a series of shear walls work as a system that is able to withstand earthquake forces. The beam also makes the working structure stiff and can absorb energy due to the very high rigidity of the coupling beam with shear walls behaving like two free cantilevers. Coupling beam can be mounted in a shear force from one wall to another so that large structural deformations can be removed. follows :

Design of this building used regulations from the Indonesian National standart as

- ASCE 7-10 Minimum load for building design and other structures [1]

- SNI 1727 : 2013 Minimum load for building design and other structures [2]

- SNI 1726 : 2012 Procedure of earthquake reistance design for building and nonbuilding structure [3]

- SNI 2847 : 2013 Building code requirements for structural concrete [4]

\section{RESEARCH METHODOLOGY}

In this design the method used is the analysis method in building structure modeling where the planning will use the dual system method, namely the special moment frame and special reinforced concrete shear walls. The case study method is in the form of re-planning with a model made according to the condition of building. The planning method used has stage of input, analysis and output. At the input stage will be ecplained about the structure geometry, dimension, and specifications of structural elements, the determination of the burden of working with 3-dimensional modeling. Stage of analydis include modeling the structure using ETABS 2016 software. The final stage is the output stage which discusses the adjusted detailing of the internal forces that occur in the building structure. The final result of this plan is expected to be that the structure of this building especially in the part of the shear wall stiffener called the coupling beam will be able to match the optimum dimensions and strenght of the structure. 
Figure 2. Modeling Plan

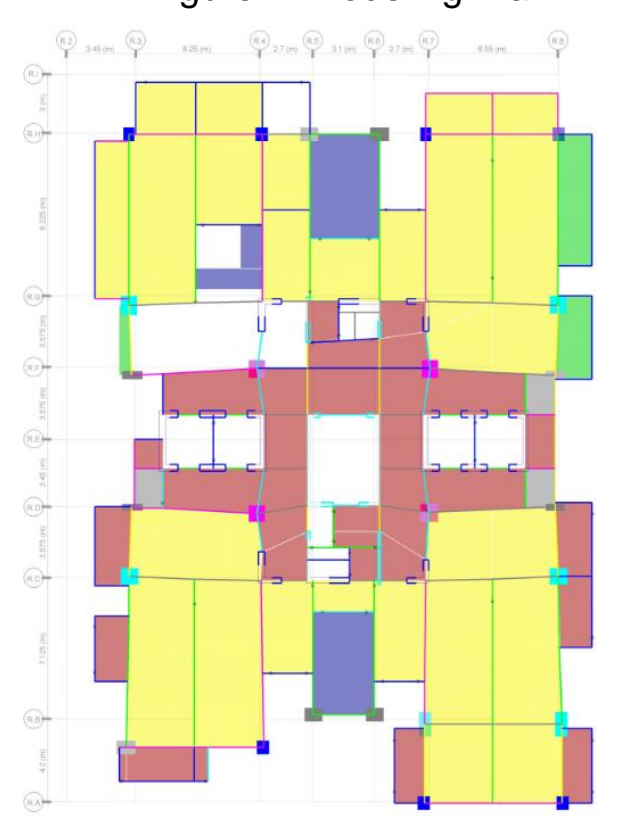

\author{
Structure Material \\ Concrete Strength : fc' $35 \mathrm{MPa}-\mathrm{fc}$ '55MPa \\ Rebar Strength : (D10 \& D13) 520MPa \\ $>\mathrm{D} 16420 \mathrm{MPa}$
}

Geometric Structure

Number of Stories : 24th floor + roof

Floor height $\quad: 4,2-4,5$ meters

Total height $\quad: 104$ meters

Function : Apartment

Location : Jakarta, Indonesia

Type of soil : Medium (Soil Type D)

Structure System

Special Moment Frame

Special Reinforced Concrete Shear Wall

Source : ETABS Modeling

\title{
RESULTS AND DISCUSSION
}

Structural Loading

The loading design follows the provisions of SNI $1727: 2013$ and ASCE 7-10 for dead load and live load, then SNI $1726: 2012$ for earthquake load.

Dead Load

Dead load consist of the weigt of all materials of construction incorporated into the building including, but not limmited to walls, floors, roofs, ceilings, starways, built-in partitions, finishes, cladding and othe similarly incorporated architectural and structural items, and fixed service equiptment including the weight of cranes.[1]

Table 1. SIDL for 1st-23th floor
\begin{tabular}{|l|c|l|}
\hline \multicolumn{1}{|c|}{ Material } & $\begin{array}{c}\text { Load } \\
\left(\mathrm{kN} / \mathrm{m}^{2}\right)\end{array}$ & \multicolumn{1}{|c|}{ Reference } \\
\hline Ceramic & 1.10 & $\begin{array}{l}\text { ASCE 7-10 Table C3-1 Ceramic or quarry tile } \\
(19 \mathrm{~mm}) \text { on 25mm mortar bed })\end{array}$ \\
\hline $\begin{array}{l}\text { Mechanical } \\
\text { Duct }\end{array}$ & 0.19 & $\begin{array}{l}\text { ASCE 7-10 Table C3-1, Mecanical Duct } \\
\text { Alowance })\end{array}$ \\
\hline $\begin{array}{l}\text { Suspended } \\
\text { Steel Channel }\end{array}$ & 0.1 & $\begin{array}{l}\text { ASCE 7-10 Tabl C3-1, Suspended Steel } \\
\text { Channel System }\end{array}$ \\
\hline $\begin{array}{l}\text { Acoustical } \\
\text { Fiber Board }\end{array}$ & 0.05 & ASCE 7-10 Table C3-1, Acoustical fiberboard \\
\hline Total & $\mathbf{1 . 4 4}$ & \\
\hline
\end{tabular}

Source : ASCE 7-10
Table 2. SIDL for roof floor

\begin{tabular}{|l|c|l|}
\hline \multicolumn{1}{|c|}{ Material } & $\begin{array}{c}\text { Load } \\
\left(\mathrm{kN} / \mathrm{m}^{2}\right)\end{array}$ & \multicolumn{1}{|c|}{ Reference } \\
\hline Waterprofing & 0.05 & $\begin{array}{l}\text { ASCE 7-10 Tabel C3-1, Waterproofing } \\
\text { Membrane Liquid Applied })\end{array}$ \\
\hline Ducting & 0.19 & $\begin{array}{l}\text { ASCE 7-10 Table C3-1, Mecanical Duct } \\
\text { Alowance) }\end{array}$ \\
\hline $\begin{array}{l}\text { Suspended } \\
\text { Steel Channel }\end{array}$ & 0.1 & $\begin{array}{l}\text { ASCE 7-10 Tabl C3-1, Suspended Steel } \\
\text { Channel System }\end{array}$ \\
\hline $\begin{array}{l}\text { Acoustical } \\
\text { fiber board }\end{array}$ & 0.05 & $\begin{array}{l}\text { ASCE 7-10 Table C3-1, Acoustical } \\
\text { fiberboard }\end{array}$ \\
\hline Total & $\mathbf{0 . 3 9}$ & \multicolumn{2}{|c|}{} \\
\hline
\end{tabular}

Source : ASCE 7-10

Live Load

A load produced by the use and occupancy of the building or other structure that does not inclued construction of environmental loads, such as earthquake load.[1]

Table 3. Live Load

\begin{tabular}{|l|c|l|}
\hline \multicolumn{1}{|c|}{ Load Type } & $\begin{array}{c}\text { Load } \\
\left(\mathrm{kN} / \mathrm{m}^{2}\right)\end{array}$ & \multicolumn{1}{|c|}{ Reference } \\
\hline Residential & 1,92 & $\begin{array}{l}\text { SNI } 1727: 2013 \text { tabel } \\
4-1\end{array}$ \\
\hline Partition & 0,72 & $\begin{array}{l}\text { SNI } 1727: 2013 \text { tabel } \\
4.32\end{array}$ \\
\hline Total & 2,64 & \\
\hline \multicolumn{3}{|c|}{ Source $:$ SNI $1727: 2013$}
\end{tabular}


Earthquake Load (Respons Spectrum Method)

Earthquake load is calculated based on SNI $1726: 2012$. Earthquake load is designed to be the probability of an earthquake of $2 \%$ within a period of 50 years or an earthquake return period of 500 years. This elastic earthquake response has $5 \%$ attenuation.[2]

- Building Locations

- Type of Soil

: Jakarta, Indonesia

- Risk Category

: Medium (SD)

Table 4 Risk category of building and other stuctures for earthquake loads

\begin{tabular}{|l|c|}
\hline \multicolumn{1}{|c|}{ Use or occupancy of Building and Structures } & $\begin{array}{c}\text { Risk } \\
\text { Category }\end{array}$ \\
\hline $\begin{array}{l}\text { All building and other structures except those listed } \\
\text { in Risk Categories I, III and IV }\end{array}$ & II \\
\hline
\end{tabular}

Source : ASCE 7-10

- Seismic Importance Factor

Table 5 Importance factors by risk category of building and other stuctures for earthquake loads

\begin{tabular}{|c|c|}
\hline Risk Category & $\begin{array}{c}\text { Seicmic Importance } \\
\text { Factor }\left(I_{e}\right)\end{array}$ \\
\hline $\mathrm{I}$ & 1.00 \\
\hline
\end{tabular}

Source : ASCE 7-10

- Seicmic design category based on short $: 0.674$

period respons acceleration parameter $\left(\mathrm{S}_{\mathrm{DS}}\right)$

- Seismic design category based on 1-S

Period respons acceleration parameter $\left(S_{D 1}\right)$

Figure 3 Respons Spectrum

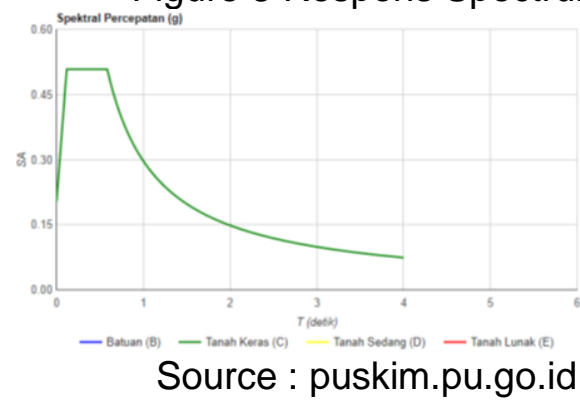

The parameter taken from

http://puskim.pu.go.id/Aplikasi/

desain spektra indonesia 2011/

- Site Class

Table 6 Site Classification

\begin{tabular}{|c|c|c|c|}
\hline Site class & $\begin{array}{c}\bar{V} s \\
(\mathrm{~m} / \text { detik) }\end{array}$ & $\bar{N}$ or $\bar{N}_{c h}$ & $\bar{S} u(\mathrm{kPa})$ \\
\hline SD (medium) & $175-50$ & $15-50$ & $50-100$ \\
\hline
\end{tabular}

Source : SNI 1726 : 2012

- $\quad$ Site Coefficient (Fa)

Table 7 Site Coefficient (Fa)

\begin{tabular}{|c|c|c|c|c|c|}
\hline $\begin{array}{c}\text { Site } \\
\text { Class }\end{array}$ & \multicolumn{5}{|c|}{$\begin{array}{c}\text { Mapped Risk-Targeted Maximum Considered } \\
\text { Earthquake (MCER) Special Respons Acceleration } \\
\text { parameter at short period }\end{array}$} \\
\hline & $S_{s} \leq$ & $S_{s}=0,5$ & $S_{s}=0,75$ & $S_{s}=1,0$ & $S_{s} \geq$ \\
& 0,25 & & 1,25 \\
\hline SD & 1,6 & 1,4 & 1,2 & 1,1 & 1,0 \\
\hline
\end{tabular}

Source : SNI 1726 : 2012

- $\quad$ Site Coefficient (Fv) 
Table 8 Site Coefficient (Fv)

\begin{tabular}{|c|c|c|c|c|c|}
\hline $\begin{array}{c}\text { Site } \\
\text { Class }\end{array}$ & \multicolumn{5}{|c|}{$\begin{array}{c}\text { Mapped Risk-Targeted Maximum Considered } \\
\text { Earthquake (MCER) Special Respons Acceleration } \\
\text { parameter at 1-s period }\end{array}$} \\
\hline & $S_{s} \leq$ & $S_{s}=0,5$ & $S_{s}=0,75$ & $S_{s}=1,0$ & $S_{s} \geq$ \\
& 0,25 & 1,25 \\
\hline SD & 1,6 & 1,4 & 1,2 & 1,1 & 1,0 \\
\hline
\end{tabular}

- Spectral response acceleration parameter at short periods $\left(S_{M S}\right)$

$\mathrm{SMS}=\mathrm{Fa} \times \mathrm{S}_{\mathrm{S}}=1,1 \times 0,674=0,7414 \mathrm{~g}$

- Spectral response acceleration parameter at a period of $1 \mathrm{~s}\left(\mathrm{~S}_{\mathrm{M} 1}\right)$

$\mathrm{SM} 1=\mathrm{Fv} \times \mathrm{S} 1=1,8 \times 0,297=0,5346 \mathrm{~g}$

- Spectral acceleration parameter at shor period $\left(S_{D S}\right)$

$\mathrm{SDS}=2 / 3 \mathrm{SMS}=2 / 3 \times 0,7414=0,494 \mathrm{~g}$

- Spectral acceleration parameter at a period of $1 \mathrm{~s}\left(\mathrm{~S}_{\mathrm{D} 1}\right)$

SD1 $=2 / 3$ SM1 $=2 / 3 \times 0,5436=0,356 \mathrm{~g}$

- Fundamental period of the structure $(T)$

$$
\begin{gathered}
T_{0}=0,2 \frac{S_{D 1}}{S_{D S}}=0,2 \frac{0,3564}{0,494}=0,144 \mathrm{~s} \\
T s=\frac{S_{D 1}}{S_{D S}}=\frac{0,3564}{0,494}=0,7214 \mathrm{~s}
\end{gathered}
$$

- $\quad$ Spectrum Acceleration (Sa)

$$
\begin{gathered}
\text { for } T<T 0, S_{a}=S_{D S}\left(0,4+0,6 \frac{T}{T_{0}}\right) \\
\text { for } T<T<T s, S_{a}=S_{D S} \\
\text { for } T \geq T s, \text { maka, } S_{a}=\frac{S_{D 1}}{T}
\end{gathered}
$$

\begin{tabular}{|c|c|c|c|c|c|c|c|c|c|}
\hline $\begin{array}{c}\text { Periode } \\
\mathrm{T} \text { (sec) }\end{array}$ & $\begin{array}{l}\mathrm{Sa} \\
(\mathrm{g})\end{array}$ & $\begin{array}{l}\text { Periode } \\
\mathrm{T} \text { (sec) }\end{array}$ & $\begin{array}{l}\mathrm{Sa} \\
(\mathrm{g})\end{array}$ & $\begin{array}{l}\text { Periode } \\
\mathrm{T}(\mathrm{sec})\end{array}$ & $\begin{array}{l}\mathrm{Sa} \\
(\mathrm{g})\end{array}$ & $\begin{array}{l}\text { Periode } \\
\mathrm{T}(\mathrm{sec})\end{array}$ & $\begin{array}{l}\mathrm{Sa} \\
\text { (g) }\end{array}$ & $\begin{array}{l}\text { Periode } \\
\mathrm{T} \text { (sec) }\end{array}$ & $\begin{array}{l}\mathrm{Sa} \\
(\mathrm{g})\end{array}$ \\
\hline 0.0 & 0.198 & 2.2 & 0.162 & 4.4 & 0.081 & 6.6 & 0.054 & 8.8 & 0.041 \\
\hline 0.2 & 0.494 & 2.4 & 0.149 & 4.6 & 0.077 & 6.8 & 0.052 & 9.0 & 0.040 \\
\hline 0.4 & 0.494 & 2.6 & 0.137 & 4.8 & 0.074 & 7.0 & 0.051 & 9.2 & 0.039 \\
\hline 0.6 & 0.494 & 2.8 & 0.127 & 5.0 & 0.071 & 7.2 & 0.050 & 9.4 & 0.038 \\
\hline 0.8 & 0.446 & 3.0 & 0.119 & 5.2 & 0.069 & 7.4 & 0.048 & 9.6 & 0.037 \\
\hline 1.0 & 0.356 & 3.2 & 0.111 & 5.4 & 0.066 & 7.6 & 0.047 & 9.8 & 0.036 \\
\hline 1.2 & 0.297 & 3.4 & 0.105 & 5.6 & 0.064 & 7.8 & 0.046 & 10 & 0.036 \\
\hline 1.4 & 0.255 & 3.6 & 0.099 & 5.8 & 0.061 & 8.0 & 0.045 & & \\
\hline 1.6 & 0.223 & 3.8 & 0.094 & 6.0 & 0.059 & 8.2 & 0.043 & & \\
\hline 1.8 & 0.198 & 4.0 & 0.089 & 6.2 & 0.057 & 8.4 & 0.042 & & \\
\hline 2.0 & 0.178 & 4.2 & 0.085 & 6.4 & 0.056 & 8.6 & 0.041 & & \\
\hline
\end{tabular}

Table 9 Spectrum Acceleration

- Seismic Design Category based on - Seismic Design Category Based

short period response acceleration parameter

Table 10 Seicmic Design Category

\begin{tabular}{|c|c|c|}
\hline \multirow{2}{*}{\begin{tabular}{c} 
Value of $S_{D S}$ \\
\cline { 2 - 3 }
\end{tabular}} & $\begin{array}{c}|c| \\
\text { I or II or } \\
\text { III }\end{array}$ & IV \\
\hline $0.33<S_{D S}<$ & C & D \\
\hline
\end{tabular}

1-S Period Response Acceleration parameter

Table 11 Seicmic Design Category

\begin{tabular}{|c|c|c|}
\hline \multirow{2}{*}{ Value of $S_{D 1}$} & \multicolumn{2}{|c|}{ Risk Category } \\
\cline { 2 - 3 } & I or II or III & IV \\
\hline $0.20 \leq S_{D S}$ & D & D \\
\hline \multicolumn{2}{|c|}{ Source : ASCE 7-10 }
\end{tabular}

Source : ASCE 7-10

- Seismic Force-Resisting System 


\begin{tabular}{|c|c|c|c|c|c|c|c|c|}
\hline \multirow[t]{2}{*}{$\begin{array}{l}\text { Seismic Force- } \\
\text { Resisting System }\end{array}$} & \multirow{2}{*}{ 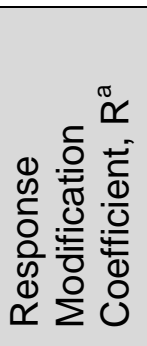 } & \multirow{2}{*}{ 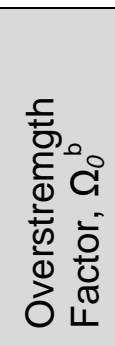 } & \multirow{2}{*}{ 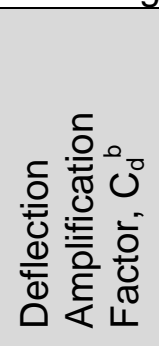 } & \multicolumn{5}{|c|}{$\begin{array}{c}\text { Structural System } \\
\text { Limitations Including } \\
\text { Structural Height } h_{n}(m)^{c} \\
\text { Limits }^{c}\end{array}$} \\
\hline & & & & B & C & $D^{d}$ & $E^{d}$ & $\mathrm{~F}^{\mathrm{e}}$ \\
\hline $\begin{array}{l}\text { Dinding Geser beton } \\
\text { bertulang khusus }\end{array}$ & $\overline{7}$ & $2 \frac{1}{2}$ & $5 \frac{1}{2}$ & $\mathrm{NL}$ & $\overline{\mathrm{NL}}$ & $\overline{\mathrm{NL}}$ & $\overline{\mathrm{NL}}$ & $\overline{\mathrm{NL}}$ \\
\hline
\end{tabular}

Load Combination

In the ultimate design, a combination of the load given to the building structure must be given in SNI 1727: 2013 article 4.2.2 with a more detailed earthquake load E of articles 7.3.4.2 and article 7.4.2. The redundancy factor must be charged to the earthquake barrier system in each of the two orthogonal directions for all structures according to this article. For structures designed for seismic design categories $D, E, F=1.3$. Get the combination given to the building, i.e. combination 1 to 18 :

Values $\rho=1.3$ and $S d s=0.494$ we get the combination of loading as follows

1. Comb. $1: 1.4 \mathrm{DL}+1.2 \mathrm{SDL}$

2. Comb. $2: 1.2 \mathrm{DL}+1.2 \mathrm{SDL}+1.6 \mathrm{LL}$

3. Comb. $3: 1.3284 \mathrm{DL}+1.3284 \mathrm{SDL}+\mathrm{LL}+0.39 \mathrm{EQx}+1.3 \mathrm{EQy}$

4. Comb. $4: 1.2692 \mathrm{DL}+1.2692 \mathrm{SDL}+\mathrm{LL}+0.39 \mathrm{EQx}-1.3 \mathrm{EQy}$

5. Comb. $5: 1.1308 \mathrm{DL}+1.1308 \mathrm{SDL}+\mathrm{LL}-0.39 \mathrm{EQx}+1.3 \mathrm{EQy}$

6. Comb. $6: 1.0716 \mathrm{DL}+1.0716 \mathrm{SDL}+\mathrm{LL}-0.39 \mathrm{EQx}-1.3 \mathrm{EQy}$

7. Comb. $7: 1.3284 \mathrm{DL}+1.3284 \mathrm{SDL}+\mathrm{LL}+1.3 \mathrm{EQx}+0.39 \mathrm{EQy}$

8. Comb. $8: 1.1308 \mathrm{DL}+1.1308 \mathrm{SDL}+\mathrm{LL}+1.3 \mathrm{EQx}-0.39 \mathrm{EQy}$

9. Comb. $9: 1.2692 \mathrm{DL}+1.2692 \mathrm{SDL}+\mathrm{LL}-1.3 \mathrm{EQx}+0.39 \mathrm{EQy}$

10. Comb. $10: 1.0716 \mathrm{DL}+1.0716 \mathrm{SDL}+\mathrm{LL}-1.3 \mathrm{EQx}-0.39 \mathrm{EQy}$

11. Comb. $11: 0.7716 \mathrm{DL}+0.7716 \mathrm{SDL}+0.39 \mathrm{EQx}+1.3 \mathrm{EQy}$

12. Comb. $12: 0.8308 \mathrm{DL}+0.8308 \mathrm{SDL}+0.39 \mathrm{EQx}-1.3 \mathrm{EQy}$

13. Comb. $13: 0.9692 \mathrm{DL}+0.9692 \mathrm{SDL}-0.39 \mathrm{EQx}+1.3 \mathrm{EQy}$

14. Comb. $14: 1.0284 \mathrm{DL}+1.0284 \mathrm{SDL}-0.39 \mathrm{EQx}-1.3 \mathrm{EQy}$

15. Comb. $15: 0.7716 \mathrm{DL}+0.7716 \mathrm{SDL}+1.3 \mathrm{EQx}+0.39 \mathrm{EQy}$

16. Comb. $16: 0.8308 \mathrm{DL}+0.8308 \mathrm{SDL}-1.3 \mathrm{EQx}+0.39 \mathrm{EQy}$

17. Comb. $17: 0.9692 \mathrm{DL}+0.9692 \mathrm{SDL}+1.3 \mathrm{EQx}-0.39 \mathrm{EQy}$

18. Comb. $18: 1.0284 \mathrm{DL}+1.0284 \mathrm{SDL}-1.3 \mathrm{EQx}-0.39 \mathrm{EQy}$

19. Gravity : 1,2 DL + 1 LL

Design Calculations

a. Calculate Weight of Building Structure (W)

The self-weight of building structures is calculated automatically by the ETABS 2016 program. The following results are the self-weight of building structures obtained:

Table 13. Calculation of Building Weight

\begin{tabular}{|c|c|c|c|}
\hline Story & Weight $(\mathrm{kg})$ & Story & $\begin{array}{c}\text { Weight } \\
(\mathrm{kg})\end{array}$ \\
\hline Storey 12 & 1176969.61 & Storey 24 & $\begin{array}{c}1273878.8 \\
5\end{array}$ \\
\hline Storey 11 & 1176969.73 & Storey 23 & $\begin{array}{c}1415795.7 \\
9\end{array}$ \\
\hline Storey 10 & 1182658.49 & Storey 22 & $\begin{array}{c}1415778.9 \\
8\end{array}$ \\
\hline Storey 9 & 1180267.77 & Storey 21 & 1243757.6 \\
\hline
\end{tabular}




\begin{tabular}{|c|c|c|c|} 
& & & 2 \\
\hline Storey 8 & 1190787.82 & Storey 20 & $\begin{array}{c}1229451.7 \\
3\end{array}$ \\
\hline Storey 7 & 1187813.88 & Storey 19 & $\begin{array}{c}1211069.5 \\
1\end{array}$ \\
\hline Storey 6 & 1190717.72 & Storey 18 & $\begin{array}{c}1119593.9 \\
5\end{array}$ \\
\hline Storey 5 & 1201990.23 & Storey 17 & $\begin{array}{c}1122199.8 \\
3\end{array}$ \\
\hline Storey 4 & 1201552.62 & Storey 16 & $\begin{array}{c}1122891.7 \\
3\end{array}$ \\
\hline Storey 3 & 1216299.05 & Storey 15 & $\begin{array}{c}1126862.7 \\
8\end{array}$ \\
\hline Storey 2 & 1216299.05 & Storey 14 & $\begin{array}{c}1151350.6 \\
2\end{array}$ \\
\hline Storey 1 & 1217896.88 & Storey 13 & $\begin{array}{c}1181081.7 \\
3\end{array}$ \\
\hline Base & - & Total & $\begin{array}{c}2831,17 \\
(\mathrm{kN})\end{array}$ \\
\hline
\end{tabular}

b. Calculating the Basic Seismic Shear Force

Calculation of basic seismic shear forces in the direction determined based on SNI 1726:2012 article 7.8.1 is determined by the equation :

$V=C_{s} . W$

Seismic Base Shear Force

Table 14 Seismic Base Shear Force

\begin{tabular}{|c|c|c|c|}
\hline \multicolumn{2}{|c|}{$C s$} & $W(\mathrm{kN})$ & $\begin{array}{c}V=C_{s} \cdot W \\
(\mathrm{kN})\end{array}$ \\
\hline$C_{s-x}$ & 0.01348 & & 3827,53 \\
\hline$C_{s-y}$ & 0.01386 & & \\
& & & 393941,17 \\
\hline
\end{tabular}

Lateral Earthquake Load Distribution

Based on SNI 1726: 2012 article 7.8.3, lateral earthquake forces (Fx) arising at all levels must be determined by the following equation:

$$
F_{x}=C_{v x} \cdot V \text { dan } C_{v x}=\frac{w_{x} h_{x}^{k}}{\sum_{i=1}^{n} w_{i} h_{i}^{k}}
$$

c. Base Shear Force Scale (Static Base Shear Comparison and Dynamic Base Shear)

Based on SNI 1726:2012 earthquake 7.9.4.1 regarding the scale of the force, this regulation implies that the dynamic basic shear force must be greater than $85 \%$ of the static shear force. Formulated as follows :

$$
\mathrm{V}_{\text {dynamic }}>0.85 \mathrm{~V}_{\text {static }}
$$

Tabel 15 Base Shear

\begin{tabular}{|c|c|c|c|c|c|}
\hline \multicolumn{2}{|c|}{ Seismic Load } & $F x(k N)$ & $F y(k N)$ & $85 \%$ Static & $\begin{array}{c}V_{\text {dynamic }}>0.85 \\
V_{\text {static }}\end{array}$ \\
\hline Static & Ex & 8804.27 & & 7483.63 & OK \\
\hline & Ey & & 9079.09 & 7717.22 & OK \\
\hline Dynamic & RSx & 906349.8 & & & \\
\hline & Rsy & & 933423 & & \\
\hline
\end{tabular}

d. Displacement 
Type of structure is a structure using a shearwall then the deviation between floors permits atea risk category II then using the formula:

$$
\Delta_{\mathrm{a}} \quad=0.020 \times h_{x}
$$

Following are the displacement data from ETABS 2016 :

Table 16 Storey Displacement

\begin{tabular}{|c|c|c|c|c|c|c|c|}
\hline Story & Elevation & $\mathrm{X}$-Dir & Y-Dir & Story & Elevation & X-Dir & Y-Dir \\
\hline $\begin{array}{c}\text { Storey } \\
12\end{array}$ & 51.2 & 4.422 & 6.263 & $\begin{array}{c}\text { Storey } \\
24\end{array}$ & 104 & 9.277 & 14.777 \\
\hline $\begin{array}{c}\text { Storey } \\
11\end{array}$ & 47.0 & 3.972 & 5.579 & $\begin{array}{c}\text { Storey } \\
23\end{array}$ & 99.2 & 9.092 & 14.303 \\
\hline $\begin{array}{c}\text { Storey } \\
10\end{array}$ & 42.8 & 3.528 & 4.907 & $\begin{array}{c}\text { Storey } \\
22\end{array}$ & 94.4 & 8.846 & 13.762 \\
\hline Storey 9 & 38.6 & 3.098 & 4.259 & $\begin{array}{c}\text { Storey } \\
21\end{array}$ & 89.6 & 8.262 & 12.471 \\
\hline Storey 8 & 34.4 & 2.683 & 3.638 & $\begin{array}{c}\text { Storey } \\
20\end{array}$ & 84.8 & 7.882 & 11.797 \\
\hline Storey 7 & 30.2 & 2.275 & 3.037 & $\begin{array}{c}\text { Storey } \\
19\end{array}$ & 80.6 & 7.515 & 11.164 \\
\hline Storey 6 & 26.0 & 1.872 & 2.456 & $\begin{array}{c}\text { Storey } \\
18\end{array}$ & 76.4 & 7.113 & 10.494 \\
\hline Storey 5 & 21.8 & 1.483 & 1.906 & $\begin{array}{c}\text { Storey } \\
17\end{array}$ & 72.2 & 6.683 & 9.794 \\
\hline Storey 4 & 17.6 & 1.113 & 1.393 & $\begin{array}{c}\text { Storey } \\
16\end{array}$ & 68 & 6.23 & 9.073 \\
\hline Storey 3 & 13.4 & 0.762 & 0.925 & $\begin{array}{c}\text { Storey } \\
15\end{array}$ & 63.8 & 5.771 & 8.353 \\
\hline Storey 2 & 9.2 & 0.439 & 0.514 & $\begin{array}{c}\text { Storey } \\
14\end{array}$ & 59.6 & 5.313 & 7.643 \\
\hline Storey 1 & 5.0 & 0.166 & 0.187 & $\begin{array}{c}\text { Storey } \\
13\end{array}$ & 55.4 & 4.87 & 6.952 \\
\hline Base & 0.0 & 0 & 0 & & & & \\
\hline
\end{tabular}

\section{Coupling Beam Calculation}

Coupling beam with $\ln / \mathrm{h} \geq 4$ must satisfy the requirement for flextural member of special moment frames, if it can be shown that the beam has adequate lateral stability. Two intersecting groups of diagonally-placed bars symmetrical about the midspan are required for deep coupling beam $(\mathrm{In} / \mathrm{h}<2)$ with a factored shear force $V u$ greater than $0,33 \lambda A c w / f c$ ', unless it can be shown otherwise that safety and stability are not compormised.Expertiment have shown that diagonally oriented reinforcement is effective only if the bars can be place at a large inclination. Two options are given for coupling beams that are not gorverned by 21.9.7.1 or 29.9.7.2 : two intersection groups of diagonally placed bars syemmetrical about the midspan may be provided or the beam can be reinforced according to the requirement of 21.5.2 through 21.5.4 (21.9.7.3) 
- Properties Coupling Beam

Table 17 Properties Coupling Beam Type C

\begin{tabular}{|c|c|c|c|c|c|c|c|c|}
\hline \multirow{3}{*}{ Storey } & \multicolumn{3}{|c|}{ Material Strength } & \multicolumn{6}{|c|}{ Dimension } \\
\cline { 2 - 10 } & $f^{\prime}$ & fy & fys & $\mathrm{b}$ & $\mathrm{h}$ & $\mathrm{Ln}$ & $\mathrm{In} / \mathrm{h}$ & $\begin{array}{c}\mathrm{d}=\mathrm{h}- \\
0,5\end{array}$ \\
\cline { 2 - 9 } & $\mathrm{MPa}$ & $\mathrm{MPa}$ & $\mathrm{MPa}$ & $\mathrm{mm}$ & $\mathrm{mm}$ & $\mathrm{mm}$ & & $\mathrm{mm}$ \\
\hline 1 5th & 55 & 420 & 520 & 400 & 1600 & 1100 & 0.7 & 1550 \\
\hline 6 10th & 55 & 420 & 520 & 400 & 1600 & 1100 & 0.7 & 1550 \\
\hline 11 15th & 55 & 420 & 520 & 400 & 1600 & 1100 & 0.7 & 1550 \\
\hline 16 19th & 45 & 420 & 520 & 300 & 1600 & 1100 & 0.7 & 1550 \\
\hline 20th & 45 & 420 & 520 & 300 & 1600 & 1100 & 0.7 & 1550 \\
\hline 21th & 45 & 420 & 520 & 300 & 1600 & 1100 & 0.7 & 1550 \\
\hline 22 23th & 35 & 420 & 520 & 300 & 1600 & 1100 & 0.7 & 1550 \\
\hline 24th & 35 & 420 & 520 & 300 & 1600 & 1100 & 0.7 & 1550 \\
\hline
\end{tabular}

- Analysis of Coupling Beam Reinforcement Type C

In determining the type of reinforcement that will be installed on the beam coupling, the provisions are used If the value of $\mathrm{Ln} / \mathrm{h}<2$ and $\mathrm{Vu}>0.33 \mathrm{Vfc}$..Acw, then the beam coupling is automatically repeated with a diagonal reinforcement, this is done only checking on the beam coupling that has value of $\mathrm{Ln} / \mathrm{h}<2$

Table 18 Checking Beam Coupling Design Type

\begin{tabular}{|c|c|c|c|c|c|c|c|c|}
\hline \multirow{3}{*}{ Storey } & $\begin{array}{l}\text { Concrete } \\
\text { Strength }\end{array}$ & \multicolumn{4}{|c|}{ Dimension } & \multirow{2}{*}{$\begin{array}{c}\text { Vu } \\
\text { Maks }\end{array}$} & \multirow{2}{*}{$\begin{array}{c}0,33 \sqrt{ } \mathrm{fc}^{\prime} \\
. \mathrm{Acw}\end{array}$} & \multirow{3}{*}{\begin{tabular}{|c|} 
Cek \\
Design
\end{tabular}} \\
\hline & $\mathrm{fc}^{\prime}$ & $b$ & $\mathrm{~h}$ & Ln & $\ln / \mathrm{h}$ & & & \\
\hline & $\mathrm{MPa}$ & $\mathrm{mm}$ & $\mathrm{nm}$ & $\mathrm{mm}$ & & $\mathrm{kN}$ & $\mathrm{kN}$ & \\
\hline 1 5th & 55 & 400 & 1600 & 1100 & 2 & 59.56 & 1566.30 & Tipe C \\
\hline $6 \sim 1$ & 55 & & & & 2 & & & Tipe C \\
\hline $11 \sim$ & 55 & 40 & 0 & & 2 & & 121 & Tipe C \\
\hline 16 19th & 45 & 300 & 1600 & & 2 & 348.54 & 1216.77 & Tipe C \\
\hline 20th & 45 & 400 & 1600 & & 2 & 287.51 & 937.11 & Tipe C \\
\hline 21th & 45 & 300 & 1600 & 1100 & 2 & 293.84 & 937.11 & Tipe C \\
\hline 22 23th & 35 & 400 & 1600 & 1100 & 2 & 254.56 & 937.11 & Tipe C \\
\hline 24th & 35 & 300 & 600 & 1100 & 2 & 108.96 & 1249.48 & Tipe C \\
\hline
\end{tabular}

Figure 4 Inner Force from ETABS 2016

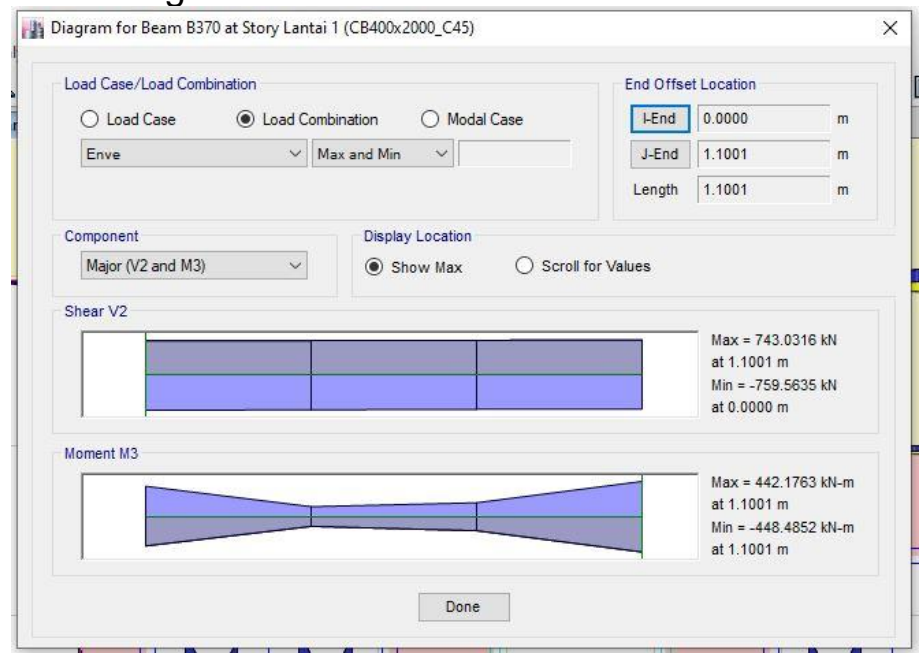

- Analysis of Reinforcement Needs Calculation

Based on the results of the force on ETABS, taken from the 1st floor type $\mathrm{S} 1$ obtained $\mathrm{Vu}=$ $759.56 \mathrm{kN}$. Therefore, the coupling beam needs diagonal reinforcement. if $\mathrm{In} / \mathrm{h}>2$ because 
of conditions $<2$ and $>4$. And if $\frac{V u}{\lambda \sqrt{f c^{\prime}} \cdot A c w}>0.33$ then calculated using $\frac{V u}{b . h . V f c^{\prime}}$. Calculate $\frac{V u}{\emptyset}$ as the following :

$$
\frac{V u}{\emptyset}=\frac{759.56}{0.75}=1012.75 \mathrm{kN}
$$

Calculating $\mathrm{Vn}$ must be calculated with the following formula in accordance with that written in SNI 1726-2012 chapter 21.9.7.4. To find out that $\mathrm{Vn}$ min and $\mathrm{Vn}$ max.

then $\mathrm{Vn}>\mathrm{Vu} / \varnothing \ldots \mathrm{OK}$ !

$$
\begin{aligned}
V n & =0.83 \sqrt{f c^{\prime} A c w} \\
& =124577.44 \mathrm{kN}
\end{aligned}
$$

- $\quad$ Vertical and Horizontal Components $\mathrm{C}$ dan T

$\sin \alpha=\frac{0.8 \times h}{\sqrt{l^{2}+(0.8 h)^{2}}}=0,758($ shear $) \quad \cos \alpha=\frac{L}{\sqrt{l^{2}+(0.8 h)^{2}}}=0,652($ flex $)$

- Diagonal Reinforcement for Shear

If all shear formed on the beam is designed to be fully borne by a diagonal reinforcement, it will be calculated with SNI chapter 21.9.7.4

$$
\begin{gathered}
V n=2 A v d . f y . \sin \alpha \\
A v d=\frac{\left(\frac{V u}{\emptyset}\right)}{2 f y \cdot \sin \alpha}=1589.70 \mathrm{~mm} 2 \\
A v d==\frac{A v d}{\frac{1}{4} \pi d^{2}}=\frac{1589.70}{\frac{1}{4} \cdot 3,14.25^{2}}=3.240 \sim 4 D 25 \\
4 \quad x \frac{1}{4} \times 3.14 \times 25^{2}=1962.50 \mathrm{~mm}^{2}>1589.70 \mathrm{~mm}^{2} \ldots(o k !)
\end{gathered}
$$

- Transversal Reinforcement Requirement

Transversal spacing must be provided for the cross section of the whole beam that meets at 21.9.7.4. Following are the steps for determining the transverse reinforcement in the design of a coupling beam with full cross section full restraints

$$
\text { : }
$$

$\checkmark$ Tranverse reinforcement area :

Because the restraining area is the entire cross section of the beam, there is no minimum dimension for determining the gross area used in determining transverse reinforcement. It is known that the coupling beam has a height of 1600 and a width of $400 \mathrm{~mm}$ with blankets on each side of $20 \mathrm{~mm}$. The diameter of the longitudinal reinforcement used is D13 and for transverse reinforcement it is assumed to require D16 reinforcement. So that parameters can be determined determining transverse reinforcement as follows:

$$
\begin{gathered}
\text { Clearance Height }=1600-(2 \times 40)=1520 \mathrm{~mm} \\
\text { Clearance width }=400-(2 \times 40)=320 \mathrm{~mm} \\
A_{g}=400 \times 600=640000 \mathrm{~mm}^{2} \\
A_{c h}=360 \times 560=486400 \mathrm{~mm}^{2}
\end{gathered}
$$

This value is then used in calculating the transverse reinforcement requirements in chapter 21.6.4.2.

$$
\begin{gathered}
\text { Condition 1: } \\
\begin{array}{c}
\frac{A_{s h}}{s}=0.3 \frac{b_{c} f_{c}^{\prime}}{f_{y t}}\left[\frac{A_{g}}{A_{c h}}-1\right] \\
\frac{A_{s h}}{s}=3.21 \mathrm{~mm}^{2} / \mathrm{mm} \\
\text { Condition } 2: \\
\frac{A_{s h}}{s}=0.09 \frac{b_{c} f_{c}^{\prime}}{f_{y t}}
\end{array}
\end{gathered}
$$




$$
\frac{A_{s h}}{s}=3.05 \mathrm{~mm}^{2} / \mathrm{mm}
$$

Of the two conditions, the largest value is used $=3.21 \mathrm{~mm} 2 / \mathrm{mm}$. With the minimum distance between diagonal reinforcement $(\mathrm{hx})$ is $200 \mathrm{~mm}$, we can determine the value of the transverse reinforcement spacing.

$\checkmark$ Transversal Spacing of chapter 21.6.4.3 (c):

$$
\begin{gathered}
100 \mathrm{~mm} \leq S_{o} \leq 150 \mathrm{~mm} \\
s_{o}=100+\left(\frac{350-h_{x}}{3}\right) \\
s_{o}=150 \mathrm{~mm}
\end{gathered}
$$

$\checkmark \quad$ Minimum Transversal Reinforcement Spacing

$$
\begin{gathered}
s_{\min 1}=6 \text { diameter diagonal }=6 \times 25=150 \mathrm{~mm} \\
s_{\min 2}=150 \mathrm{~mm}
\end{gathered}
$$

Longitudinal spacing between $150 \mathrm{~mm}$ transverse reinforcement is taken. From this transverse reinforcement space, it can be determined the need for transverse reinforcement

$$
\begin{gathered}
\text { requriement area }=s \times \frac{A_{s h}}{S} \\
\text { requirement area }=150 \times 3,21=480.97 \mathrm{~mm}^{2}
\end{gathered}
$$

Mounted transverse reinforcement 4 feet D13, with an area of $530.66 \mathrm{~mm} 2$.

From the determination of transverse reinforcement is the installation of 4-foot D13-150mm reinforcement which curbs the entire cross section of the coupling beam.

- Confinement Reinforcement Requirements

Confinement reinforcement calculations will be carried out as follows for the $x$ and $y$ directions

$$
\begin{gathered}
A_{s h}=0.3\left(\frac{b_{c x} f_{c}^{\prime} s}{f_{y}}\right)\left(\frac{A_{g}}{A_{c h}}-1\right) \\
A_{s h}=545.49 \mathrm{~mm}^{2}
\end{gathered}
$$

$A_{s h}$ min that must be installed for the $x$ direction is $545.49 \mathrm{~mm} 2$. So for Ash to be installed $A_{s h}$ $=5.0 .25 . \pi \cdot d^{2}=3 \times 0.25 \times 3.14 \times 13^{2}=663.352 \mathrm{~mm}^{2}$. So that is attached to the $\times 5 \mathrm{D} 13$ direction hook

$$
\begin{gathered}
A_{s h}=0.3\left(\frac{b_{c y} f_{c}^{\prime}}{f_{y}}\right)\left(\frac{A_{g}}{A_{c h}}-1\right) s \\
A_{s h}=761.54 m^{2}
\end{gathered}
$$

$A_{\text {sh }}$ min which must be installed for $y$ direction is $761.54 \mathrm{~mm} 2$. So for Ash to be installed A_sh $=5.0 .25 \cdot \pi \cdot d^{\wedge} 2=4 \times 0.25 \times 3.14 \times 13^{\wedge} 2=816.4 \mathrm{~mm}^{\wedge} 2$. So that will be attached to the $x$ 5D13 direction hook

- Reinforcement for Longitudinal

Calculations for longitudinal reinforcement are as follows:

$$
\begin{gathered}
\emptyset M_{n}=\left(\emptyset \times \mathrm{A}_{\mathrm{vd}} x f_{y} x \cos \alpha\right) x(h-100) / 10^{6} \\
\emptyset M_{n}=683.61 \mathrm{kNm}
\end{gathered}
$$

Based on the results of the moment on ETABS, taken from the 1st floor type A obtained $\mathrm{Mu}$ (etabs) $=448.49 \mathrm{kNm}$. And to calculate the need for longitudinal reinforcement as follows $A_{s} \min =\frac{0.25 x \sqrt{f_{c}^{\prime}}}{f_{y}} b_{w} d$ and may not be smaller than $A_{s} \min =\frac{1.4}{f_{y}} b_{w} d$, so :

$$
\begin{gathered}
A_{s} \min =\frac{0.25}{f_{y}} b_{w} d=\frac{0.25}{420} \cdot 400.52 .5=2205 \mathrm{~mm}^{2} \\
A_{s}=5 * \frac{1}{4} * \pi * d^{2}=2 * \frac{1}{4} * 3.14 * 25^{2}=2453.13 \mathrm{~mm}^{2}
\end{gathered}
$$

So for As that will be used 5D25

Coupling Beam Reinforcement 
Table 19 Coupling Beam Reinforcement

\begin{tabular}{|c|c|c|c|c|c|c|c|c|c|c|}
\hline \multirow{2}{*}{ Storey } & \multicolumn{2}{|c|}{ Diagonal } & \multicolumn{3}{|c|}{ Transversal } & \multicolumn{3}{c|}{ Confinement } & Longitudinal \\
\cline { 2 - 12 } & qty & $\begin{array}{c}\text { Dia } \\
(\mathrm{mm})\end{array}$ & $\begin{array}{c}\text { Dia } \\
(\mathrm{mm})\end{array}$ & qty & $\begin{array}{c}\text { spacing } \\
(\mathrm{mm})\end{array}$ & $\begin{array}{c}\text { Dia } \\
(\mathrm{mm})\end{array}$ & qty & $\begin{array}{c}\text { spacing } \\
(\mathrm{mm})\end{array}$ & qty & $\begin{array}{c}\text { Dia } \\
(\mathrm{mm})\end{array}$ \\
\hline 1 5th & 4 & $\mathrm{D} 25$ & $\mathrm{D} 13$ & 4 & 150 & $\mathrm{D} 13$ & 5 & 150 & 5 & $\mathrm{D} 25$ \\
\hline 6 10th & 3 & $\mathrm{D} 25$ & $\mathrm{D} 13$ & 4 & 150 & $\mathrm{D} 13$ & 5 & 150 & 5 & $\mathrm{D} 25$ \\
\hline 11 15th & 3 & $\mathrm{D} 25$ & $\mathrm{D} 13$ & 3 & 150 & $\mathrm{D} 13$ & 4 & 150 & 5 & $\mathrm{D} 25$ \\
\hline 16 19th & 2 & $\mathrm{D} 25$ & $\mathrm{D} 13$ & 3 & 150 & $\mathrm{D} 13$ & 4 & 150 & 5 & $\mathrm{D} 25$ \\
\hline 20th & 2 & $\mathrm{D} 25$ & $\mathrm{D} 13$ & 3 & 150 & $\mathrm{D} 13$ & 4 & 150 & 5 & $\mathrm{D} 25$ \\
\hline 21th & 2 & $\mathrm{D} 25$ & $\mathrm{D} 13$ & 3 & 150 & $\mathrm{D} 13$ & 3 & 150 & 4 & $\mathrm{D} 25$ \\
\hline 22 23th & 2 & $\mathrm{D} 25$ & $\mathrm{D} 13$ & 3 & 150 & $\mathrm{D} 13$ & 3 & 150 & 4 & $\mathrm{D} 25$ \\
\hline 24th & 2 & $\mathrm{D} 25$ & $\mathrm{D} 13$ & 3 & 150 & $\mathrm{D} 13$ & 4 & 150 & 4 & $\mathrm{D} 25$ \\
\hline
\end{tabular}

Figure 5 Schematic of mounting beam coupling reinforcement

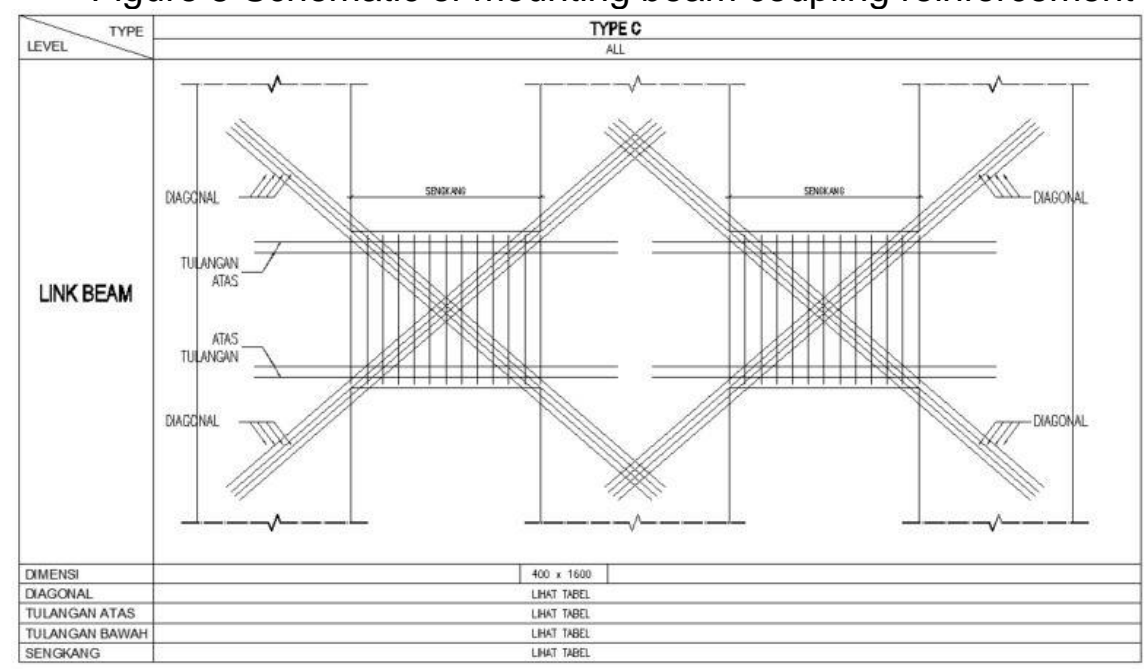

\section{CONCLUSIONS}

Based on the results of the design of the Residence building structure in the The Jakarta Stature project with a focus on the beam coupling with the special moment bearing frame system and the sliding wall system the following conclusions can be drawn :

a. Earthquake Analysis (structural properties of earthquakes) between designs is as follows::

\section{$\checkmark \quad$ Natural Periode}

The natural period of the design structure modeled by ETABS meets the requirements with a maximum limit according to SNI 1726: 2012 article 7.8.2 :

$$
\begin{array}{lll}
T_{a}\left(T_{\text {min }}\right) & =0.0488 \times 104^{0.9} & =3.1897 \mathrm{~s} \\
T_{\text {maks }} & =1.4 \times 3.1897 & =4.4656 \mathrm{~s}
\end{array}
$$

Tabel 20 Periode Struktur Out Put Etabs

\begin{tabular}{|c|c|c|c|}
\hline & $T_{x}$ & $T_{y}$ & Note \\
\hline \hline Design & $3,773 \mathrm{~s}$ & $3.699 \mathrm{~s}$ & OK \\
\hline
\end{tabular}




\section{$\checkmark \quad$ Displacement (Story Drift)}

All designs have fulfilled the permit deviation specified in SNI 1726: 2012 article 7.8.6 where the largest deviation value is on the 24th floor with direction value $x=9.277$ and $\mathrm{y}$ direction $=14.77 \mathrm{~mm}$

\section{b. Beam Coupling Dimensions and Reinforcement Details}

From the analysis results obtained calculation of reinforcement requirements for TYPE C beam coupling which has been mentioned in the previous chapter that this type of beam coupling will be adjusted due to design clashes with the needs of mechanical and architectural work, therefore according to the results of this Type $\mathrm{C}$ coupling beam analysis can be adjusted to a new dimension with a height of $1600 \mathrm{~mm}$ from the previous $2000 \mathrm{~mm}$.

\section{Suggestions}

Based on the results that have been studied can be given suggestions, including:

- In planning this structure using the burdens of the Indonesian National Standard (SNI). When analyzing the structure to be more accurate, it is recommended to use the burdens that have been imposed on the structure design by the planner (consultant).

- To get the optimum design and avoid the design clash with other disciplines in structural planning, it needs to be considered in more detail, especially for needs that must meet the minimum specifications.

- In planning this coupling beam only designed using diagonal reinforcement, for further research the authors suggest that in similar cases the beam coupling is designed with a strut and tie model so that a comparison of steel tonnage between the two methods will be obtained.

\section{REFERENCES}

[1] ASCE, (2010). Minimum Design Loads for Building and Other Structures. American Society of Civil Engineers, USA.

[2] Badan Standarisasi Nasional,(2013). Beban Minimum untuk Perancangan Bangunan Gedung dan Struktur Lain (SNI 1727:2013). SNI, Jakarta

[3] Badan Standarisasi Nasional,(2018).Tata Cara Perencanaan Ketahanan Gempa Untuk Struktur Bangunan Gedung dan Non Gedung (SNI 1726:2012).

[4] Badan Standarisasi Nasional,(2013). Persyaratan Beton Struktural untuk Bangunan Gedung (SNI 2847:2013). SNI, Jakarta

[5] Bambang B.,(2017). Contoh Desain Bangunan Tahan Gempa Dengan Sistem Rangka Pemikul Momen Khusus dan Sistem Dinding Struktur Khusus Di Jakarta. Bandung:Penerbit ITB

[6] Reshma C.,(2014).Comparative Study on Solid and Coupled Shear Wall. Kerala:IJCIET (International Journal of Civil Engineering and Technology)

[7] Hasibuan, Muhammad Khoirun, Khadavi, Rini Mulyani,(2017). Perencanaan Struktur Gedung Rusunawa Dengan Sistem Ganda Yaitu Kombinasi Antara Sistem Rangka Pemikul Momen Khusus (SRPMK) Dengan Sistem Dinding Shearwall (SDS). Padang:Universitas Bung Hatta.

[8] Hasibuan, Romi Cornelus,(2012). Perencanaan Dinding Geser Berpasangan (Coupled Shearwall) Pada Struktur Bangunan Bertingkat Tinggi. Yogyakarta:Universitas Atma Jaya Yogyakarta

[9] Imran, Iswandi. Fajar Hendrik,(2016). Perencanaan Lanjut Struktur Beton Bertulang. ITB Press

[10] Krismahardi, Widi. Pupuk Wahyuono,(2017). Perencanaan Struktur Gedung "Sunter Park View Apartment" Sunter-Jakarta Utara. Semarang:Universitas Diponegoro

[11] Muhammad Annas, Ferry.(2017). Kapasitas Geser Balok Kopel dengan Perkuatan Tulangan Pada Area Diagonal Tekan. Surakarta:Universitas Muhammadiyah Surakarta.

[12] Pamungkas, Anugrah. Erny Harianti.(2018). Struktur Beton Bertulang Tahan Gempa. 


\section{Yogyakarta : Penerbit Andi}

[13] Poudel, Ashwin. Dkk.(2018). Diagonally Reinforced Concrete Coupling Beams : Effects of Axial Restraint.Kansas:The University Of Kansas Center For Research, INC.

[14] Sharma, Abhisek.Prince Sharma. Seismic Performance Evaluation of Hybrid Coupled Shear Wall in High Rise Buildings.(2019) : IJITEE (International Journal of Innovative Technology and Exploring Engineering)

[15] Shin, Dong-IK, Muhammad Haroon, Jung-Yoon Lee.(2017). Shear Behaviour Of Reinforced Concrete Coupling Beams Predicted Using Nonlinear Finite Element Analysis. Seoul:Sung Kyun Kwan University

[16] Subha.(2018). Seismic Analysis of Hybrid Coupled Shear Wall System with GFRP Coupling Beams.Kerala:NSS College of Engineering

[17] Tavio, Usman Wijaya.(2018).Desain Rekayasa Gempa Berbasis Kinerja (Performance Based Design). Yogyakarta : Penerbit Andi

[18] Widiati, lis Roin.Ilmi Sidik Rabrusun.(2016). Perbandingan Sistem Rangka Pemikul Momen Khusus Dengan Sistem Ganda. Papua : Universitas Yapis Papua 